\title{
Estimation of protective indices in chicken vaccinated with single and booster doses of trivalent salmonella vaccine
}

\begin{abstract}
Background: Infection with Salmonella species is a major health concern for human and animals on a global scale. Most cases of Salmonellosis results in complicated diarrhea, elderly and immune-compromised persons can be at risk for more severe invasive infections which can be life threatening. Control of Salmonellosis in poultry by vaccination is a possible means of controlling the problems.
\end{abstract}

Material and method: Two different inactivated trivalent $S$. Enteritidis, $S$. Typhimurium and S. Kentucky vaccine batches of 2 different origins were used to vaccinate salmonella free chickens with either single dose or single then booster dose vaccination programs. These chickens were reared in clean separated pens and later on were challenged with virulent $S$. Enteritidis, S. Typhimurium and S. Kentucky virulent strains 3 weeks post single or booster doses. Then protective indices was estimated as mean of vaccine evaluation.

Results: Vaccinated birds showed varied protection according to challenge strains, vaccination program and origin of vaccine. Protective indices was estimated as $71 \%$ and $66.2 \%$ for local and commercial inactivated trivalent salmonella vaccine respectively when chicken challenged 3 weeks post single dose vaccination. Protective indices raised up to $82.5 \%$ and $79.5 \%$ respectively when birds challenged 3 weeks post booster vaccination.

Conclusion: Evaluation of the combined salmonella vaccine depending on protective indices is more obvious and the picture more better than evaluation depending on either measurement of humoral response or mortalities post challenge because of protective indices depends on several parameters reflecting the immune status of the birds including mortalities, clinical signs and post mortem lesions.

Keywords: salmonella vaccine, salmonellosis, Samonella enteritides, Salmonella typhimurium,
Volume 6 Issue 3 - 2018

Selim S Salama, Fatema El zahraa Gamal, Fatema Gadallah, Eman Solima, Nourhan Nagy

Central Laboratory for Evaluation of Veterinary Biologics (CLEVB), Abbasia, Egypt

Correspondence: Selim S Salama, Central Laboratory for Evaluation of Veterinary Biologics (CLEVB), El-Sekka Elbeeda St.,Abbasia, PO box I3I, Post code I I38I, Cairo, Egypt, Tel 00201066655085, Email selimsalama2000@yahoo.com

Received: May 14, 2018 | Published: June 04, 2018

\section{Introduction}

Salmonellosis is one of the most common food borne bacterial disease in the world, the great majority of salmonella infections in humans are food borne with Samonella enteritides and Salmonella Typhimurium accounting for a major part of the problem. ${ }^{1}$ The genus Salmonella consists of more than 2500 serovares $^{2}$ it is reported that approximately $60 \%$ of human salmonellosis were caused by $S$. typhimurium well as Salmonella Enteritidis. ${ }^{3}$

The global epidemiology of non typhoidal salmonella disease is complex, with diverse serovars in different regions worldwide, bosing a substantial challenge for vaccine development. Salmonella typhurium and Salmonella enteritidis have been the most prevalent serovars in people and animals for many years. (anglo)Human cases of salmonellosis were found to be caused by $S$. shubra, S. enteritidis, $S$. typhimurium and $S$. kentucky were reported to be wide spread in a study carried out. ${ }^{4}$ Salmonella enterica is a major global food-born pathogen causing life threatening infection.

Infection with Salmonella species is a major health concern for human and animals on a global scale. Although most cases of
Salmonellosis results in complicated diarrhea. Elderly and immunecompromised persons can be at risk for more severe invasive infections which can be life threatening and may be require antimicrobial therapy American pediatric association. Constructed three bivalent vaccines for preventing both Salmonella typhimurium and Salmonella new port infections and concluded that the delivery of heterologous antigen is a prospective approach for developing salmonella vaccines. ${ }^{5}$

Control of Salmonella infections in poultry is posing itself as one of the difficult problems not only for those who are concerned with poultry industry, but also for public health hazard because of the fact that the most of serovars of Salmonella harbored by poultry can act as potential pathogens for man. ${ }^{6}$ Killed and live attenuated products have been used for controlling salmonella in poultry production and vaccination with live attenuated products has proved to be effective. ${ }^{7}$ Prevention of avian salmonellosis using inactivated vaccine has been reported by several authors to provide good protection with decrease or absence of the residual virulence. ${ }^{8}$ So, this present work aimed to evaluate the challenge vaccination assay which reflects protection against (S. enteritidis, S. typhimurium and) by S. kentucky estimating the protective indices. 


\section{Material and methods}

\section{Vaccine used}

Two different batches of combined trivalent inactivated Salmonella enteritidis, Salmonella typhimurium, Salmonella kentucky were supplied by CLEVB (Central Laboratory for Evaluation of Veterinary Biologics) and used in the evaluation study. The first one represents a local producer (Veterinary Serum and Vaccine Research Institute) while the second one represents a commercial imported vaccine.

\section{Bacterial strain}

Local field isolates of $S$. enteritis, S. typhimurium and S. kentucky were kindly obtained from (CLEVB). These strains were used in challenge processes.

\section{Vaccination -challenge assay}

Vaccination program: 570 Salmonella free chickens were reared in clean separated pens and fed on balanced ration. These chickens were divided into 3 groups as following:

a. First group: It consists of 240 chickens and it was subdivided into 2 equal subgroups, first sub group was vaccinated with locally produced trivalent Salmonella vaccine while the second sub group was vaccinated: With the commercial trivalent Salmonella vaccine (120 chicken for each).

b. Second group It comprising of 240 chicken and subdivided into 2 equal subgroups (120 each), first sub group was vaccinated with locally produced trivalent Salmonella vaccine while the second sub group was vaccinated with commercial trivalent salmonella vaccine, after 3 weeks, the subgroups were boostered doses from corresponding vaccines.

c. Third group: It was comprising of 90 chicken and was subdivided equally in parallel to other groups kept as control groups.

Vaccination assay: Each group of the two subgroups in the first main group was subdivided into 3 groups each subgroup was challenged intramuscularly with $0.1 \mathrm{ml} \mathrm{I/M}$ rout of one of $S$. enteritidis $1 \times 10^{7} \mathrm{CFU} /$ bird, S. typhimurium $1 \times 10^{6} \mathrm{CFU} /$ bird or $S$. kentucky $1 \times 10^{7} \mathrm{CFU} / \mathrm{bird}$ 3 weeks post single dose vaccination in parallel to 15 unvaccinated control chicken for each.
The same treatment was adapted for the vaccinated chicken in the second main group but was challenged 3 weeks post booster dose vaccination.

Calculating the protective indices using the following formula by, ${ }^{7}$ protective indices (PIs) were assessed according to mortality and PM lesions (PML)

$P I=\frac{\%(M \& P M L) \text { controls }-\% \text { vaccinated }(M \& P M L)}{\% \operatorname{control}(M \& P M L)} \mathrm{X} 100$

\section{Results}

As regarding to the protection obtained in vaccinated chicken after challenge with Salmonella enteritidis strain, Table 1 showed that protection obtained Post single dose was $(72.5 \%)$ while it raised up to $(81.25 \%) 3$ weeks post booster vaccination with combined trivalent salmonella vaccine while it was only $(13.3 \%)$ in the non vaccinated chicken group.

As shown in Table 2 the protective indices achieved with virulent Salmonella typhimurium strain challenge after vaccination with trivalent salmonella vaccine was $(71.25 \%)$ and $(81.25 \%)$ after single dose and booster dose respectively while it was $(6.6 \%)$ in the control non vaccinated chicken group.

As regards to protection obtained in the vaccinated chicken after challenge with virulent Salmonella Kentucky strain, Table 3 showed that vaccinated chicken groups with trivalent Salmonella vaccine showed marked protection level more than the control vaccinated chicken. Overall protection after challenging with virulent Salmonella kentucky strain were $(72.5 \%)$ after single dose vaccination which raised up to $(81.25 \%)$ after booster dose, while it was only $(13.3 \%)$ in the non vaccinated control group.

As shown in Table 4, protective indices were estimated for challenged chicken post either single dose or booster dose vaccination. Protective indices were 71 and 66.5 for the 2 different local and commercial tested vaccine batches respectively. While it was 82.5 and 79.5 in case of challenge post booster dose of vaccination assay for both tested vaccines respectively.

Table I Results of vaccination challenge assay against Salmonella enteritidis 3 weeks post single and booster dose vaccination

\begin{tabular}{lllllllll}
\hline \multicolumn{2}{l}{ Booster dose vaccination } & \multicolumn{5}{c}{ Single dose vaccination } \\
\cline { 1 - 3 } control & Total & Imported & Local & control & Total & Imported & Local \\
\hline 15 & 80 & 40 & 40 & 15 & 80 & 40 & 40 & No \\
6 & 7 & 4 & 3 & 6 & 10 & 6 & 4 & $\mathrm{M}$ \\
7 & 8 & 4 & 4 & 7 & 12 & 6 & 6 & $\mathrm{~S}$ \\
13 & 15 & 8 & 7 & 13 & 22 & 12 & 10 & $\mathrm{~T} / \mathrm{A}$ \\
12 & 15 & 8 & 7 & 12 & 20 & 10 & 10 & $\mathrm{R}$ \\
$13.30 \%$ & $81.25 \%$ & $80 \%$ & $82.50 \%$ & $13.30 \%$ & $72.50 \%$ & $70 \%$ & $75 \%$ & $\mathrm{P} \%$ \\
\hline
\end{tabular}

No, Number of birds; M, Mortalities; S, Symptoms; T/A, Total affected; R, Re isolation; P\%, Protection\% 
Table 2 Results of vaccination challenge assay against Salmonella typhimurium weeks post single and booster dose vaccination

\begin{tabular}{|c|c|c|c|c|c|c|c|c|}
\hline \multirow[b]{2}{*}{ control } & \multirow[b]{2}{*}{ Total } & \multicolumn{3}{|c|}{ Booster dose vaccination } & \multicolumn{3}{|c|}{ Single dose vaccination } & \\
\hline & & Imported & Local & control & Total & Imported & Local & \\
\hline 15 & 80 & 40 & 40 & 15 & 80 & 40 & 40 & $\mathrm{~N}$ \\
\hline 7 & 7 & 4 & 3 & 7 & 12 & 6 & 6 & $M$ \\
\hline 7 & 9 & 5 & 4 & 7 & 11 & 6 & 5 & S \\
\hline 14 & 15 & 9 & 7 & 14 & 23 & 12 & 11 & T/A \\
\hline 12 & 13 & 9 & 7 & 13 & 23 & 12 & 11 & $\mathrm{R}$ \\
\hline $6.60 \%$ & $81.25 \%$ & $77.50 \%$ & $82.50 \%$ & $6.60 \%$ & $71.25 \%$ & $70 \%$ & $72.50 \%$ & $\mathrm{P} \%$ \\
\hline
\end{tabular}

No, Number of birds; M, Mortalities; S, Symptoms; T/A, Total affected; R, Re isolation; P\%, Protection\%

Table 3 Results of vaccination challenge assay against Salmonella Kentucky weeks post single and booster dose vaccination

\begin{tabular}{|c|c|c|c|c|c|c|c|c|}
\hline \multirow[b]{2}{*}{ Control } & \multirow[b]{2}{*}{ Total } & \multicolumn{3}{|c|}{ Booster dose vaccination } & \multicolumn{3}{|c|}{ Single dose vaccination } & \\
\hline & & Imported & Local & control & Total & Imported & Local & \\
\hline 15 & 80 & 40 & 40 & 15 & 80 & 40 & 40 & $\mathrm{~N}$ \\
\hline 6 & 6 & 3 & 3 & 6 & 9 & 5 & 4 & $M$ \\
\hline 7 & 9 & 5 & 4 & 7 & 13 & 7 & 6 & $\mathrm{~S}$ \\
\hline 13 & 15 & 8 & 7 & 13 & 22 & 12 & 10 & T/A \\
\hline 12 & 14 & 7 & 7 & 13 & 22 & 12 & 10 & $\mathrm{R}$ \\
\hline $13.3 \%$ & $81.25 \%$ & $80 \%$ & $82.5 \%$ & $13.3 \%$ & $72.5 \%$ & $70 \%$ & $75 \%$ & $\mathrm{P} \%$ \\
\hline
\end{tabular}

No, Number of birds; M, Mortalities; S, Symptoms; T/A, Total affected; R, Re isolation; P\%, Protection\%

Table 4 Estimated protective indices post single and booster dose vaccination for local and commercial vaccines

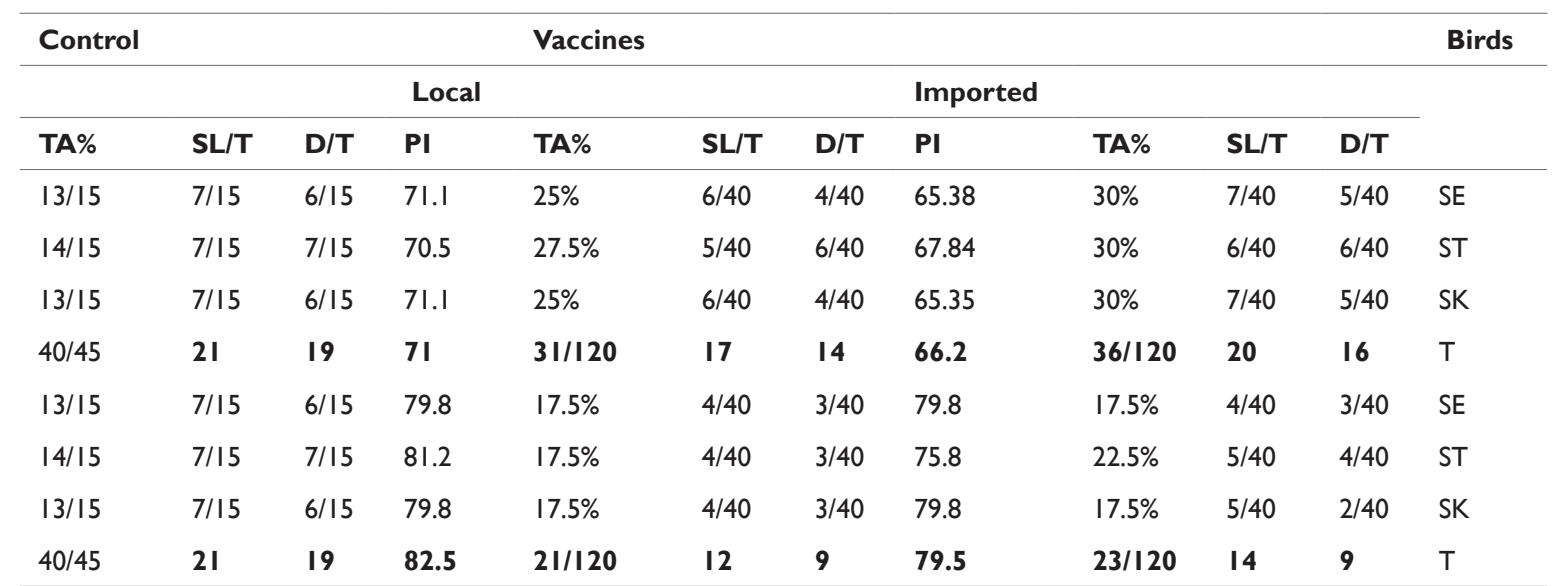

\section{Discussion}

Inactivated trivalent salmonella vaccine which composed of $S$ enteritidis, S. typhimurium and S. kentucky now is one of the target vaccines that is used in the control of the most common salmonellae in between poultry and human public health.

As regarding to the protection obtained in the vaccinated chickens after challenge with virulent salmonellae post single or booster doses, evaluation of the protective value of the used trivalent salmonella vaccines using challenge test was used according to. ${ }^{9}$ This test is considered the master test for determination of the protective value of a vaccine. ${ }^{8}$
The obtained data showed that the vaccinated chickens gave protection of $72.5 \%$ when challenged with virulent $S$. enteritidis strain 3 weeks post single dose vaccination. This protection was raised to $81.25 \%$ when birds were challenged 3 weeks post boostering as shown in Table 1. Also chickens showed protection of $71.25 \%$ when challenged with virulent $S$. typhimurium strain 3 weeks post single dose vaccination which raised to $81.25 \%$ when challenged 3 weeks post boostering as shown in Table 2. Vaccinated Birds gave protection of $72.5 \%$ when challenged with virulent S. Kentucky strain 3 weeks post single dose vaccination meanwhile this protection was raised to $81.25 \%$ when birds were challenged 3 weeks post boostering as shown in Table 3 . The achieved protection values by the 3 vaccine batches are accepted to pass the vaccine for use according to ${ }^{10}$ These 
results are nearly in are agree with the results which were obtained by ${ }^{10-12}$ immunized chickens with Salmonella typhimurium vaccine then subsequently challenged with the virulent strain and concluded that the vaccinated birds showed resistance to infection with virulent Salmonella typhimurium and this was reflected in bacterial infection and shedding. Also ${ }^{13}$ confirmed that vaccinated birds with killed Salmonella enteritidis vaccine can protect them from challenge with virulent organisms at the end of 2 nd week post booster dose. ${ }^{8}$ Stated that challenge test is the master test for the determination of Salmonella kentucky vaccine and ${ }^{14}$ reported that the protective value against virulent Salmonella kentucky post challenge in chickens vaccinated with Salmonella kentucky vaccine was $80 \%$ and the achieved protection value by the used vaccine was accepted to pass the vaccine for use according to ${ }^{15,10}$.

Concerning the overall protective indices for the vaccinated chickens, it was $71 \%$ in case of local vaccine, $66.2 \%$ in case of commercial vaccine as demonstrated in Table 4 when chickens were challenged after single dose vaccination assay. At the same concerns, challenged birds after booster dose vaccination assay revealed a protective indices of $82.5 \%$, and $79.5 \%$ corresponding to local and commercial imported batches respectively as shown in Table 4 . This observation confirmed that the trivalent Salmonella vaccine could be more effective and more protective against natural and experimental infections with different types of salmonellae included in the vaccine preparation. Also the combination of the three different salmonellae either Salmonella kentucky, Salmonella typhimurium or Salmonella enteritidis does not interfere or affect the immune response of birds against each of them. ${ }^{8}$

\section{Conclusion}

Evaluation of the combined salmonella vaccine depending on protective indices is more obvious and the picture is better than evaluation depending on either measurement of humoral response or mortalities post challenge because of protective indices depends on several parameters reflecting the immune status of the birds including mortalities, clinical signs and post mortem lesions.

The trivalent Salmonella inactivated vaccine is vital in eliciting adequate antibody titers which aiding in conferring adequate immunity enable birds to pass experimental and so natural infections with the salmonella species. Also, the combination of Salmonella typhimurium, Salmonella enteritidis and Salmonella Kentucky vaccine proved to be of great value because of its high efficacy with a good and significant result in safety and protection.

Vaccination with the locally prepared polyvalent Salmonella inactivated vaccine could contribute protection and lower Salmonella prevalence in layers, breeders and in turn in broiler chickens.

\section{Acknowledgements}

None.

\section{Conflict of interest}

Author declares that there is no conflict of interest.

\section{References}

1. OIE. Terrestrial Animal Health Code. Chapter 6.5.Prevention, Detection and Control of Salmonella in poultry. 2017.

2. Bell C, Kyriakides A. Salmonella. In: Blackburn C, McClure P, editors. Food borne pathogens, hazards, risk analysis and control. CRC Press LLC; 2002. p. 307-331.

3. CDC. Increases in fluoroquinolone- resistant Neisseria gonorrhoeaeHawaii and California. MMWR Morb Mortal Wkly Rep. 2002;51(46):10411044.

4. Angelo KM. Chu A, Anand M, et al. Outbreak of Salmonella Newport infections linked to cucmbers united states, 2014. Morb Mortal Wkly Rep. 2015;64:144-147.

5. Galanis E1, Lo Fo Wong DM, Patrick ME, et al. Web-based surveillance and global Salmonella distribution, 2000-2002. Emerg Infect Dis. 2006;12(3):381-388.

6. Xinxin Z, Qinlong D, Renyong J. Front cell infection Microbial. Resarch Center of Avian Disease. College of veterinary Medicine, Sichuan Agricultural Univerisity, Chengdu, China; 2017.

7. Van Duijkeren E, Wannet WJ, Houwers DJ, et al. Serotype and phage type distribution of Salmonella strains isolated from humans, cattle, pigs and chicken in the Netherland from 1984 to 2001. J Clin Microbial. 2004;40(11):3980-3985.

8. Timms LM, Marshall RN MF, Breslin. Laboratory assessment of protection given by an experimental Salmonella Enteritidis PT4 inactivated adjuvanted vaccine. Vet Rec. 1990;127(25-26):22-29.

9. Paiva JB, Penha FRAC, Arguello YMS, et al. Efficacy of several Salmonella vaccination programs against experimental challenge with Salmonella gallinarum in commercial browen layer and broiler breeder hens. Brazil J of Poult Sci. 2009;11(1):65-72.

10. Egyptian standard Regulation for evaluation of veterinary biologics. CLEVB; 2009.

11. Mona I El-Enbaawy, Zakia AM Ahmed, Sadek MA, et al. Protective efficacy of Salmonella local strains representing groups B, in a prepared polyvalent formalin Inactivated Oil Adjuvant Vaccine in Layers. Microbiological Research. 2013;4(4):288-295.

12. Muir WI, Bryden WL, Husband AJ. Evaluation of the efficacy of intraperitoneal immunization in reducing Salmonella Typhimurium infection in chickens. Poultry Science. 1998;77(12):1874-1883.

13. Meenakshi M, Bakshi CS, Butchaiah G, et al. Adjuvanated outer membrane protein vaccine protects poultry against infection with Salmonella Enteritidis. Veterinary Research Communication. 1999;23(2):81-90.

14. Nourhan N, Sadek MA, Wafaa R, et al. Efficacy of locally prepared Salmonella Kentucky vaccine in chicken. Benha veterinary medical journal. 2015;29(2):153-160.

15. Heddleston KL. Pasteurellosis: isolation and identification of avian athogens. Am Assoc Avian Patho. 1975:38-51. 